\title{
El tamizaje oportunista de fibrilación auricular no aumentó la tasa de detección en el ámbito de la atención primaria
}

Opportunistic screening for atrial fibrillation did no increase the detection rate in primary care setting

\section{Comentado de:}

Uittenbogaart SB, et al. BMJ2020;370:m3208. PMID: $32938633^{1}$

\section{Objetivo}

Investigar si el screening o tamizaje oportunista (sólo la detección de pacientes que visitaron la práctica) en atención primaria aumenta la detección de fibrilación auricular (FA) en comparación con la atención habitual.

\section{Diseño \\ Ensayo controlado aleatorizado por conglomerados.}

\section{Lugar y participantes}

Se compararon 47 prácticas de detección y 49 prácticas de atención primaria habitual en los Países Bajos. El estudio fue abierto y se llevó a cabo entre septiembre de 2015 y agosto de 2018. En cada práctica se seleccionaron de manera aleatoria 200 pacientes de 65 años o más, sin antecedentes conocidos de FA en el sistema de historia clínica electrónica. En el grupo de intención de detección se incluyeron 9.218 pacientes elegibles para realizar la práctica, $55,0 \%$ de los cuales eran mujeres, con una edad media de 75,2 años. En el grupo de atención habitual, 9.526 pacientes fueron elegibles para el tamizaje, $54,3 \%$ de ellos de sexo femenino, con una edad media de 75,0 años.

\section{Intervenciones}

El tamizaje oportunista (aquel realizado en pacientes que acudían a su consulta general) consistió en la realización de tres pruebas índice: palpación del pulso, medición electrónica de la presión arterial con un algoritmo de FA y electrocardiografía (ECG) con un dispositivo electrocardiográfico de mano de una sola derivación. El estándar de referencia fue el ECG de 12 derivaciones, realizado en pacientes con al menos una prueba índice positiva y en una muestra de pacientes $(10 \%)$ con las tres pruebas negativas. Si el ECG de 12 derivaciones no mostraba FA, se invitaba a los pacientes a realizar más pruebas de detección mediante una monitorización continua con un electrocardiógrafo Holter durante dos semanas.

\section{Principales medidas de resultado}

Diferencia en la tasa de detección de FA recién diagnosticada durante un año en las prácticas de atención por intención de detección frente a las habituales.

\section{Resultados}

El seguimiento se completó para 8.874 pacientes en las prácticas de intención de detección y para 9.102 pacientes en las prácticas de atención habituales. Se encontraron 144 (1,62\%) nuevos diagnósticos de FA en el grupo de intención de detección frente a $139(1,53 \%)$ en el grupo de atención habitual (odds ratio [OR] ajustado 1,06; intervalo de confianza [IC] del $95 \% 0,84$ a $1,35)$. De 9.218 pacientes elegibles en el grupo de intención de detección, $4.106(44,5 \%)$ participaron en el protocolo de tamizaje. En estos pacientes, el ECG de 12 derivaciones detectó FA de nuevo diagnóstico en 26 pacientes $(0,63 \%)$. En los 266 pacientes que continuaron con la monitorización Holter, se encontraron cuatro diagnósticos más de FA.

\section{Conclusiones}

El tamizaje oportunista de FA en pacientes de atención primaria de 65 años o más, no aumentó la tasa de detección de FA, lo que implicaría que esta práctica no es útil en ese ámbito.

\footnotetext{
Fuente de financiamiento/Conflicto de interés de los autores: El proyecto fue financiado por ZonMw (la Organización Holandesa para Investigación y Desarrollo) y por los Centros Médicos de las Universidades de Amsterdam. Microlife/Retomed proveyó el equipamiento sin cargo, empresa que no tuvo ningún rol en el diseño del estudio, la recolección, el análisis o la interpretación de los datos, ni la decisión de publicar el artículo. Los autores declararon haber recibido el apoyo de las entidades mencionadas para este trabajo, sin haber tenido relaciones financieras con organizaciones $u$ otras actividades que pudieran considerarse conflictos de interés.
}

\section{Comentario}

La detección temprana de la FA es importante para garantizar su tratamiento rápido y adecuado. No solo tiene como objetivo controlar los síntomas, sino también evitar complicaciones ${ }^{2}$ : prevenir los eventos vasculares cerebrales (ACV), el inicio posterior de síntomas, el remodelado eléctrico y mecánico, la taqui-miocardiopatía, los trastornos mentales y la morbi-mortalidad relacionada con la FA, y las hospitalizaciones. Sin embargo, esta práctica conlleva el riesgo de generar ansiedad en el paciente y conducir a falsos positivos que conduzcan a estudios o tratamientos innecesarios.

Muchos episodios son asintomáticos o paroxísticos, lo que dificulta su detección. Las personas con FA asintomática detectada por examen clínico tienen un riesgo similar de ACV que otros pacientes con $\mathrm{FA}^{2-4}$.

Los resultados de este estudio difieren de otros estudios como el SAFE ${ }^{5}$ donde se detectaron más casos de FA mediante el tamizaje. Una de las razones que comentan los autores para la falta de aumento en la detección de FA es la menor edad de los pacientes que participaron del estudio, ya que la incidencia de la FA depende de la edad ${ }^{6}$. La alta prevalencia de FA en los centros participantes (10\%) habla de que la detección en la práctica habitual ya es elevada en los Países Bajos. La selección de una muestra aleatoria de pacientes de atención primaria aumentó la generalizabilidad de los hallazgos, pero también significó que una proporción de los pacientes seleccionados no fueran elegibles para realizar el tamizaje. Se documentó que los pacientes que participaron en la detección oportunista eran más jóvenes (con menor riesgo de FA) y con menos comorbilidades que los pacientes que no participaron. 
Igualmente, el rastreo oportunista podría ser aplicado en países donde existe un porcentaje mayor de FA desconocida. Las guías europeas del año $2020^{7}$ recomiendan la detección oportunista para FA por toma de pulsos periféricos o realización de ECG en pacientes de edad mayor o igual a 65 años (recomendación clase I, con nivel de evidencia B) y el tamizaje sistemático con ECG en pacientes con edad mayor o igual a 75 años o con alto riesgo de ACV (recomendación clase Ila, con nivel de evidencia B).

\section{Conclusiones del comentador}

El tamizaje oportunista de FA en el ámbito de la atención primaria de la salud sigue siendo un tema controvertido. Quizás aplicado en una población seleccionada, con mayor riesgo de presentar este trastorno, y con nuevas tecnologías que faciliten un monitoreo más prolongado y repetitivo, se observen mayores beneficios.

Gustavo Maid [ Servicio de Cardiología, Sección Electrofisiología, Hospital Italiano de Buenos Aires. gustavo.maid@hospitalitaliano.org.ar ]

Maid G. El tamizaje oportunista de fibrilación auricular no aumentó la tasa de detección en el ámbito de la atención primaria. Evid Actual Pract Ambul. 2020;23(4):e002105. Comentado de: Uittenbogaart SB, et al. Opportunistic screening versus usual care for detection of atrial fibrillation in primary care: cluster randomised controlled trial. BMJ2020;370:m3208. PMID: 32938633

\section{Referencias}

1. Uittenbogaart SB, van Gurp NV, Lucassen WAM, et al. Opportunistic screening versus usual care for detection of atrial fibrillation in primary care: cluster randomised controlled trial. BMJ. 2020;370:m3208. Available from: 10.1136/bmj.m3208.

2. Boriani G, Laroche C, Diemberger I, et al. Asymptomatic Atrial Fibrillation: Clinical Correlates, Management, and Outcomes in the EORP-AF Pilot General Registry. m J Med. 2015;128(5):509-518.e2. Available from: 10.1016/j.amjmed.2014.11.026.

3. Potpara TS, Polovina MM, Marinkovic JM, et al. A comparison of clinical characteristics and long-term prognosis in asymptomatic and symptomatic patients with first-diagnosed atrial fibrillation: The Belgrade Atrial Fibrillation Study. Int J Cardiol. 2013;168(5):4744-4749. Available from: 10.1016/j. ijcard.2013.07.234.

4. Siontis KC, Gersh BJ, Killian JM, et al. Typical, atypical, and asymptomatic presentations of new-onset atrial fibrillation in the community: Characteristics and prognostic implications. Heart Rhythm. 2016;13(7):1418-1424. Available from: 10.1016/j.hrthm.2016.03.003.

5. Fitzmaurice DA, Richard-Hobbs FD, Jowett S, et al. Screening versus routine practice in detection of atrial fibrillation in patients aged 65 or over: cluster randomised controlled trial. BMJ. 2007;335(7616):383-383. Available from: 10.1136/bmj.39280.660567.55.

6. Lowres N, Olivier J, Chao TF, et al. Estimated stroke risk, yield, and number needed to screen for atrial fibrillation detected through single time screening: a multicountry patient-level meta-analysis of 141,220 screened individuals. PLoS Med. 2019;16:1002903-1002906. Available from: 10.1371/journal.pmed. 1002903.

7. Hindricks G, Potpara T, Dagres N, et al. 2020 ESC Guidelines for the diagnosis and management of atrial fibrillation developed in collaboration with the European Association of Cardio-Thoracic Surgery (EACTS). Eur Heart J. 2020;p. ehaa612. Available from: 0.1093/eurheartj/ehaa612. 